\title{
Proposed Framework for the Assessment of Business Intelligence Platforms for Medium-to-Large Hospitals Using Hierarchical Decision Modeling and Expert Judgments
}

\author{
Nadeem U. Shahid, Nasir J. Sheikh \\ Technology Management Department, The University of Bridgeport, Bridgeport, USA \\ Email: nshahid@my.bridgeport.edu
}

How to cite this paper: Shahid, N. U., \& Sheikh, N. J. (2022). Proposed Framework for the Assessment of Business Intelligence Platforms for Medium-to-Large Hospitals Using Hierarchical Decision Modeling and Expert Judgments. Open Journal of Business and Management, 10, 525-542. https://doi.org/10.4236/ojbm.2022.101029

Received: December 12, 2021

Accepted: January 27, 2022

Published: January 30, 2022

Copyright $\odot 2022$ by author(s) and Scientific Research Publishing Inc. This work is licensed under the Creative Commons Attribution International License (CC BY 4.0).

http://creativecommons.org/licenses/by/4.0/

(c) (i) Open Access

\begin{abstract}
This research focuses on the assessment of business intelligence platforms for medium-to-large hospitals using hierarchical decision modeling methodology (HDM) and expert judgment. The healthcare data come from different source systems, like EMRs or HR software, to different departments, like radiology or pharmacy. The data comes from all over the organization. Healthcare data is complex and complicated, i.e., the data is structured and unstructured and has various formats like text, numeric, images, multimedia, paper, etc. Even before the pandemic, the healthcare data was growing. However, healthcare data has grown exponentially during the pandemic, resulting in a growing need for storage, retrieval, and analysis challenges using the traditional approaches. In addition, there is a growing demand for clinical and administrative information from hospital executives and services providers. The use of business intelligence (BI) is seen as a possible solution to this challenge. BI helps direct, high-level decision-making by evaluating practice performance and predicting patient outcome trends. BI application/use has the potential to reduce costs and build healthier revenue streams, and at the same time, deliver the best patient care. BI can assist decision-makers from clinic to billing, $\mathrm{HR}$, and everything in between. The objective of the current research paper is to help healthcare executives in the assessment of BI alternatives by considering multiple criteria and multiple stakeholders. The research applied a multi-criteria approach, considered People, Business Processes, and Technology perspectives, and used Hierarchical Decision Modeling (HDM) methodology to evaluate solution alternatives. HDM methodology requires the expert judgment of experts to provide the relative ranking of the perspectives and criteria. There are four alternative solutions suggested. Three solutions
\end{abstract}


are cloud-based, and one is legacy, aka traditional in-house solution. Cloud and legacy systems will co-exist for the long run.

\section{Keywords}

Hierarchical Decision Modeling (HDM), Assessment of Business Intelligence Solutions, Business Intelligence Cloud Platforms

\section{Background}

Healthcare organizations have diverse data sources; among many challenges, combining the data for a single view of the patient is the most daunting task. In the meantime, due to pandemic data, growth is very high compared to other sectors. Today, approximately $30 \%$ of the world's data volume comes from the healthcare industry. By 2025, the compound annual growth rate of data for healthcare will reach $36 \%$. That is $6 \%$ faster than manufacturing, $10 \%$ faster than financial services, and $11 \%$ faster than media \& entertainment (RBC Capital Markets, 2021). The volume, variety, velocity, and variety of data create many challenges for the healthcare sector. Data storage and analyzing the data are the most common.

In a 1958 article, IBM researcher Hans Peter Luhn used business intelligence. He defined intelligence as: "the ability to apprehend the interrelationships of presented facts in such a way as to guide action towards a desired goal." Over the past few decades, the term business intelligence has gone through many transformations. The present-day definition of business intelligence was evolved in 1960 from decision support systems. Decision support systems, data warehouses, executive information systems, OLAP, and business intelligence systems gained popularity starting in the 1980s Elena, C. (2011). Business Intelligence (BI) is a broad term that covers reporting, dashboarding, OLAP, data mining, data warehouse, tools, and practices (Olszak \& Ziemba, 2007; Mettler \& Vimarlund, 2009).

Among the many other BI systems functions, one function integrates data from various sources, primarily from internal information systems, with data from outside the organization, e.g., statistical, financial, and demographic/census data from multiple data sources. These systems provide adequate, timely, reliable, and up-to-date information on different aspects of enterprise activities (Kakkanatt et al., 2018). BI is also defined as the capability of an enterprise to collect, maintain, and organize knowledge, reflecting the magnitude and impact of data-related problems faced by today's businesses (Kao et al., 2016).

According to Research and Markets (2020), the cloud computing industry has seen rapid growth; due to flexibility, low cost, rapid deployment, and scalability, businesses are transitioning to cloud computing. According to the cloud computing source, the expected revenue for the year 2025 is reaching US $\$ 832.1$ billion compared to the year 2020 , which was US\$374.1 billion. 
The current study will add the taxonomy of assessment of business intelligence platforms, an assessment model with multiple perspectives for mid-large hospital decision-makers. In addition, the study first time applies the Hierarchal Decision Model (use of multi-criteria decision-making) in identifying BI platform solutions (Figure 1).

National Institute of Standards and Technology's (NIST) standard SP 800-145 defines cloud computing as "a model for enabling ubiquitous, convenient, on-demand network access to a shared pool of configurable computing resources (e.g., networks, servers, storage, applications, and services) that can be rapidly provisioned and released with minimal management effort or service provider interaction.”

Scalability, flexibility, processing power, availability, and less operational cost make cloud computing one of the fastest-growing technologies in the computer industry, benefiting all types of enterprises (Taleb \& Mohamed, 2020). However, when selecting a cloud service model for delivering the cloud-based solution, the right decision will impact the organization for many years to come. Therefore, it is equally important to understand each service model's capabilities, the responsibilities of the service provider, and the responsibilities of cloud service consumers.

In research from Precedence Research (2021), the global healthcare cloud computing market size was valued at USD 22.43 billion in 2019 and projected to register a compound annual growth rate of around $17.8 \%$ during the projection time from 2020 to 2027. Currently, the US is the leading player in healthcare cloud computing. Cloud computing is the on-demand delivery of IT resources over the Internet with pay-as-you-go pricing. There are four cloud models: public, private, hybrid, and Community. In addition, three services models are Infrastructure as a Service (IaaS), Platform as a Service (PaaS), and Software as a Service (SaaS), are considered for the study.

Public clouds are the most common and popular type of cloud computing. The cloud resources (like servers and storage) are owned and operated by a third-party cloud service provider and delivered over the Internet. The advantages are lower costs, no maintenance, scalability, and high reliability (Microsoft, 2021).

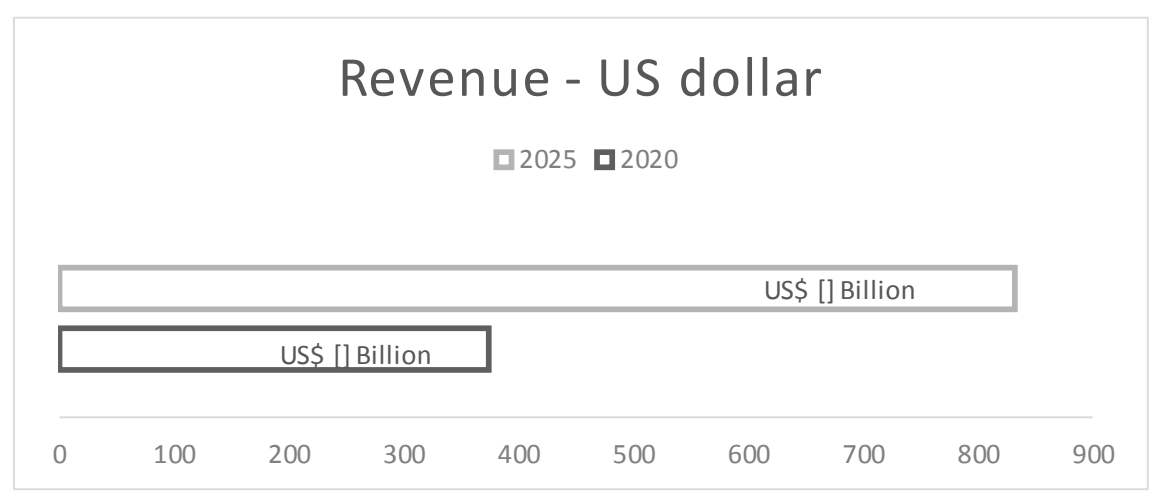

Figure 1. Growth in cloud computing industry (Source: Research and Markets, 2020). 
A private cloud consists of cloud computing resources used exclusively and usually physically located at organization's or a third-party service provider can host it. The hardware and software are dedicated solely to the organization. The services and infrastructure are always maintained on a private network. As a result, the private cloud is more flexible, and organizations have more control and scalability (Microsoft, 2021).

A hybrid cloud is a type of cloud computing that combines a private cloud-with a public cloud. Hybrid clouds allow data and apps to move between the two environments. Hybrid cloud is the choice of many organizations where compliance, regulatory, and data privacy are the core of the business. Hybrid cloud computing enables businesses to keep sensitive data in their data centers yet scale the computing resources (Microsoft, 2021). The advantages are that businesses have control over sensitive information, it is flexible, easy to use, and cost-effective solution as pay for the extra computing as needed.

Infrastructure as a Service (IaaS), Platform as a Service (PaaS), and Software as a Service (SaaS) are the three most popular cloud service models. These models are interchangeably referred to as cloud service models or cloud computing service models. The term "As a Service" is slightly different from traditional IT infrastructure. The organization pays or leases IT assets for the business's day-to-day activities. Instead, the cloud service provider owns, manages, and maintains the assets; the customer consumes them via an Internet connection. Usually, a consumer pays for them on a subscription or pay-as-you-go basis.

IaaS is on-demand access to cloud-hosted physical and virtual servers, storage, and networking-the back-end IT infrastructure for running applications and workloads in the cloud (IBM Cloud Education, 2021). The main advantage is that customers can scale up the computing resources when needed and scale down when not needed. As a result, IaaS save businesses significant upfront Capital and Maintenance expenses (Lal \& Bharadwaj, 2016). Among the many advantages are high availability, lower latency, high-level security, and can scale up and down as and when needed in almost no time. In addition, IaaS offers solutions between Virtual Machines (Vms) hosted on shared physical resources or dedicated unshared physical hardware resources (Al Hadwer et al., 2021).

PaaS is on-demand access to a complete, ready-to-use, cloud-hosted platform for developing, running, maintaining, and managing applications. The cloud service providers provide the full range of services in the platform, servers, operating systems, software, storage, networking, database, middleware, runtimes, frameworks, development tools (IBM Cloud Education, 2021). However, there is more to PaaS. For example, the PaaS service model offers the development teams faster development, testing, and production time, among the many advantages. In addition, there is low-to-no-risk if businesses want to test new applications, operating systems, or development tools, simplified collaboration, and a more scalable approach.

SaaS is on-demand access to ready-to-use, cloud-hosted application, ready-to-use 
software. Like all the cloud service models, SaaS is also paid as you go, or monthly, annual subscription service for the business (IBM Cloud Education, 2021). The cloud service providers provide all the application(s) and necessary infrastructure. The vendor's responsibility is to manage all the upgrades, updates, and patches. In addition, the cloud service provider ensures the service and data's availability, performance, and security. The advantages are minimal risk, anytime and anywhere access to data and application(s), and easy scalability.

Legacy/Proprietary in-house

Such systems could be any homegrown system, from daily extracts using any tools to statistical models or reporting. Some legacy or on-premises data warehouses or in-house reporting tools are at the core of traditional BI tools. Applications leverage third-party API handshakes and integrations with the latest licensed open-source software applications. Developing, implementing, and maintaining such legacy systems are usually on-premises. The homegrown analytics systems require significant capital investment for hardware, real estate acquisition, and a long-term project cycle plan that factors in a phased execution (Dayama, 2021).

\section{Literature Review}

The literature review is conducted to study the related body of knowledge to understand the current state of BI, trends, gaps, and case studies in the three areas mentioned above. In addition, the objective is to identify the foundation for the research, which includes the compilation of criteria and factors that make up the perspectives.

This study's objective is a BI platform focusing on the Healthcare sector and cloud service models. For the literature review, the researched study material was organized using Mendeley reference manager, which manages and shares research papers and generates bibliographies for scholarly articles. The searched terms varied across the literature. However, the search terms included: business intelligence systems, multi-criteria decision making, business intelligence application for healthcare, life cycle assessment, and clinical data warehouse.

One hundred ten journal papers, white papers, technical blogs, and industry websites were reviewed during the literature review process. Out of 110,80 were most relevant to this research based on the title and abstract analysis. Sixty-five papers were selected for detailed analysis, out of which 50 were selected for the current research paper.

The keywords searched are given in Table 1; for historical perspective, the reference is searched in academic research databases for the in-depth detail of the theory.

The focus of the current study is the technology assessment of business intelligence cloud platforms. Figure 2 gives an overview of the research intersections.

The literature review suggests that AHP has been applied inconsistently in 
Table 1. Keywords and database.

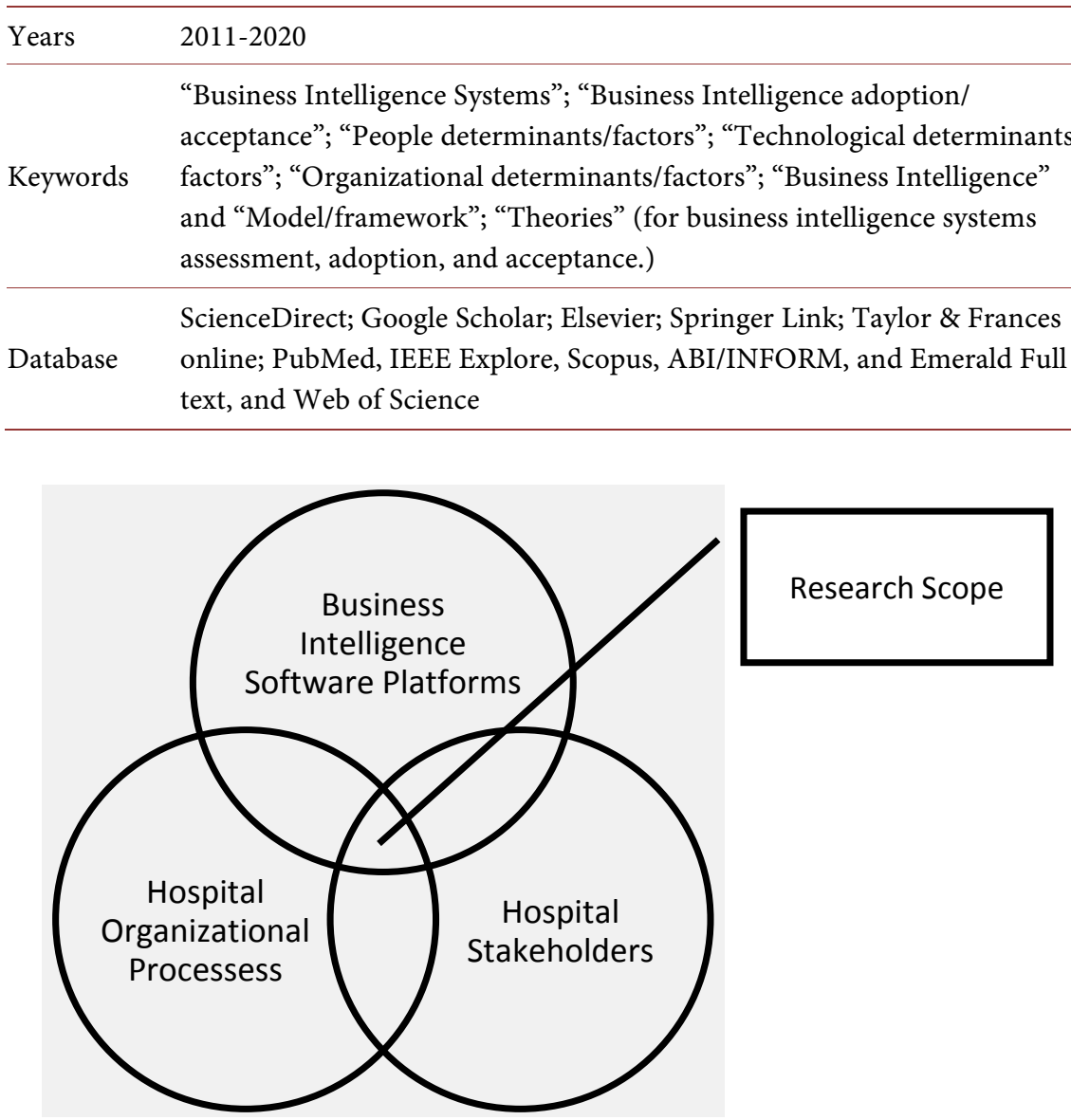

Figure 2. Related research area.

healthcare research. The specific areas where AHP methodology was applied are shared decision making, clinical guidelines, and healthcare management (Schmidt et al., 2015).

The Healthcare sector is challenged with providing healthcare services to unexpectedly vast populations due to pandemics. In addition, the sector is already under immense financial pressures, requiring better information and technology integration. Information technologies can help the healthcare sector ease the pressure. Cloud computing offers scalable and new ways to deliver information technology services for better patient outcomes (Ali et al., 2018). In research from Kumar Sharma et al. (2021), the correlation between healthcare data management and cloud computing can significantly value the healthcare sector.

Research has indicated that Business Intelligence can improve operational performance in compliance, maintain billing, collections, predict profitability, and transform information into knowledge (Simonson, 2020). Research from Sharma et al. (2014) shows that top-performing organizations rely on data analytics for day-to-day decisions and long-term strategic decision-making. Data analytics help organizations better understand the market and their customers. 
As a result, business Intelligence and analytics create value for the firm. Many research results highlight that Business Intelligence has improved the data analysis, data collection, decision-making process, financial, strategical planning, and bringing customers to the center stage of all the business processes (de Carvalho et al., 2016).

The need for better patient care, improved patient outcomes, and reduced care costs are the objectives for the healthcare sector. The BI healthcare analytics can be the technology initiative to lower the cost and improve the quality of care (Zheng et al., 2014). In research from Chang et al. (2013), BI from the process perspective intends to enhance the quality of decision making and reduce the time it takes. Bach et al. (2016) research paper are based on the technology acceptance model (TAM). The framework for investigating the determinants of business intelligence systems. Three significant factors are proposed for the acceptance model 1) motivation for an adopter to use a system, 2) perceived ease of use (PEU), and 3) perceived usefulness (PU).

Research from Serumaga-Zake (2017) indicates that the quality of the system and BI user satisfaction should be enriched to maximize the benefits of BI systems.

Undertaking the realization of BI systems in organizations, it is first necessary to determine a general vision of such systems. The systems also have to be related to business objectives. This stage involves specifying the informational needs of organizations, simultaneously paying attention to crucial IT decision-makers and specialists. Ranking informational needs (carried out based on their importance) allows for highlighting, e.g., essential indexes while realizing business strategies (Chaudhary, 2004).

In research from Banta (2009), technology is defined as "science or knowledge applied to a defined purpose." The term was first coined in 1960 in the United States. Early technology assessment was described as policy research that looks deeply into the short and long-term consequences. The very fundamental objective of technology assessment was to offer policymakers information on policy alternatives. Business intelligence and cloud service initiatives for the healthcare sector have multiple perspectives. Making the right strategic moves and identifying suitable technologies can result in competitive advantage, better patient outcomes, and cost-saving.

Multi-perspective technology assessment is the process of evaluating options considering the varied viewpoints of subject matter experts. The method of decision-making considering relevant perspectives and related criteria will result in a well-rounded solution for the long-term future of an organization. As a result, technology assessment reduces the risk of wasteful investments. The assessment of the business intelligence alternative decision model for mid-large hospitals will cover the three broad perspectives, narrowed by various related criteria.

However, the first and foremost reason for unsuccessful BI implementation lies in the truth that user and organization process requirements are not consi- 
dered when deciding the BI implementation. This framework aims to address these gaps and bring SMEs from different perspectives to reach a common consensus.

The research paper by Isik, Oyku (2009) highlights that the proper implementation of BI can benefit the enterprise in terms of improved performance, better profitability, lowering cost, and increased efficiency. However, many organizations have not achieved success with BI implementation. The current study takes a different approach by applying a BI alternative solution assessment framework. A multi-perspective assessment is applied where experts from other areas can come together and provide their input.

\section{Research Objective}

This study aims to develop a comprehensive methodology to evaluate the mid-large hospital business intelligence solutions under the three perspectives. The research will enable decision-makers from all three perspectives with many competing criteria and perspectives in a given healthcare environment-the need for Business Intelligence cloud solutions. Furthermore, the consideration of various solutions can vary depending on the needs of organizations.

\subsection{Problem Statement}

Evaluating business intelligence in mid-large hospitals using HDM and expert judgment? Is generally a complex problem since multiple perspectives and diverse experts can have disagreements. This complex decision problem is decomposed into a hierarchical decision model where different business intelligence alternatives or choices under consideration are presented to experts. The selection of various levels of criteria (or constraints) can then be applied to address the question, "In the judgment of management and experts, which alternative is preferred?" This paper addresses this problem for the mid-large hospitals during the assessment of business intelligence solution alternatives. The model will also offer in the relative rankings of the alternative alternatives.

An HDM software application, PCM (Pair-Wise Comparison Method), developed by the Department of Engineering and Technology Management at Portland State University, Portland, Oregon, is used to enter research data and derive initial results. The PCM software is based on HDM developed by Kocaoglu.

\subsection{Requirements}

- The assessment model will be organized into various hierarchical levels (People, Technology, and Organization Processes)

- The assessment model for BI solutions in the healthcare industry should provide a consistent approach to developing business intelligence, alternative solutions in healthcare. The assessment model is defined into levels of importance Perspectives, Criteria, and Sub criteria 


\subsection{Assessment Modeling Design Process}

Business Intelligence implementation in mid-large hospitals is still lagging compared to a commercial organization. In addition, the diverse nature of data sources, the volume, velocity, variety, and veracity of the data, make it more difficult. The current research will apply HDM methodology to access the alternative solutions.

The assessment model is developed by defining the objectives for the model and then criteria that would ve used to select the alternative among the listed options. The initial assessment framework is designed to understand and define the problem, and also, expert help was sought during this process. The valuable experts' input helped refine the model and finally come up with the current model, which is presented in this paper.

\section{Methodology}

As explained in the problem statement that the HDM methodology involves expert judgment and subject matter experts. HDM is the new approach compared to existing methodologies related to the decision-making process.

The HDM methodology has three parts:

1) Hierarchical decision model

2) Subject matter expert/expert panel

3) Research instrument

The HDM has the following elements:

- Mission/objectives

- Criteria

- Sub-criteria (also called factors)

- Alternatives (under consideration)

\subsection{Multi-Criteria Decision}

Multi-criteria area decision making (MCDM) is a prevalent methodology used in decision-making methodologies. MCDM can satisfy the multitude of conflicting objectives in the best way possible. Measurement in MCDM is all derived by directly comparing objects. Thomas Slaty establishes that direct comparison is necessary to establish intangible properties with no scaled-up measurement. Therefore, methods based on pairwise comparison form a significant part of MCDM.

The highlight of this discussion is about the research steps, participants selection, data collection, conversion, and validation. To summarize, the methodology is divided into three parts: 1) hierarchical decision modeling, 2) expert panel selection, and 3) research instrument (Barham \& Daim, 2020; Sheikh et al., 2016). In recent research, the decision models are proved to be an effective method in understanding the relationship between multiple factors and expert panel quantification for relative ranking. 


\subsection{Hierarchical Decision Modeling}

Kocaoglu (1983) proposed the methodology for "measuring the subject values via constant-sum comparisons." The multi-criteria decision-making method proposed and introduced by Kocaoglu helps in decision-making in the case of complex and competing factors (Barham \& Daim, 2020). In the research paper, Pereira et al. (2019) explained the constant-sum method, where experts assign 100 points between two decision variables, higher the relative importance, and higher the score. Recent research has proved that the constant sum method is more effective in expert judgment when quantifying the significance between two factors (Mudavadi et al., 2016).

HDM has been successfully applied in several studies, like Developing a Hierarchical Decision Model to Evaluate Nuclear Power Plant Alternative Siting Technologies (Lingga, 2000). Here are some examples of A hierarchical decision model (HDM) methodology applied:

Among the many studies, HDM methodology was applied to study the adoption of electronic health records (Mudavadi et al., 2016). The paper presented a model to facilitate managerial decision-making for re-manufacturing and re-use using HDM methodology (Guide \& Pentico, 2003). Hierarchical decision methodology was applied to select target markets for a new personal healthcare device (Sheikh, Kim, \& Kocaoglu, 2016). Research paper on health technology diffusion: Case of remote patient monitoring (RPM) for the care of senior population (Alanazi \& Daim, 2021). HDM methodology was applied to study the technology adoption potential of medical devices: the case of wearable sensor products for pervasive care in neurosurgery and orthopedics (Hogaboam \& Daim, 2018). Multi-Criteria Hierarchical Decision Modeling and multi-stakeholder methodology were applied to study the assessment of electronic authentication policies (Son et al., 2018). There is an extensive list of diverse research topics using HDM methodology in technology assessment-related research.

\subsection{Expert Judgment}

Expert judgment quantification provides the categorization and relative ranking of the factors that influence the selection of alternatives. There are many factors, and understanding the relationship between factors and prioritizing the factors is at the core of HDM methodology. The experience of subject matter experts, their diverse backgrounds related to $\mathrm{BI}, \mathrm{HC}$, and Technology provides valuable input to prioritize perspectives and factors.

\subsection{Pairwise Comparison}

The pairwise comparisons method was proposed by Marie Antoine Nicolas de Caritat, marquis de Condorcet (1743-1794). Over time, with research, the pairwise comparison has transformed. However, pairwise comparison is a practical methodology, and HDM is a widespread technique dealing with complex decision-making (Hou, 2016). The number of questions in the pairwise comparison 
method is given by the equation below, where $n$ is the total number of items:

Number of questions $=n(n-1) / 2$

\section{Assessment Model}

The results of the study literature resulted in the form of the following model. Figure 3 depicts the POT assessment with three perspectives and ten criteria, and thirty sub-criteria in a hierarchy.

The perspectives are the crucial considerations for the technology assessment models. It is a well-known phenomenon that perspectives impact positively or negatively. As a result of a positive impact, it enables specific technology(s) adoption. However, the negative impact can result in no adoption or sometimes challenges the survival of technology (Sheikh et al., 2016).

In the research paper from Linstone (1981), it was the first time suggested the three perspectives for technology assessment 1) Technical perspective, 2) Organizational perspective, and 3) Personal perspective. According to the Merriam-Webster dictionary, "Perspective" is the capacity to view things in their true relations or relative importance.

In this study, the People's Perspective (P perspective) is to study the internal and external stakeholders. Organizational Processes (O perspective) study hospital-specific organizational business processes, and Technology Perspective ( $\mathrm{T}$ perspective) is to study the technical elements.

All the identified perspectives are essential, but the limited scope of the research's three perspectives are considered. Each perspective can have any number of elements as criteria and sub-criteria. For example, an individual can study

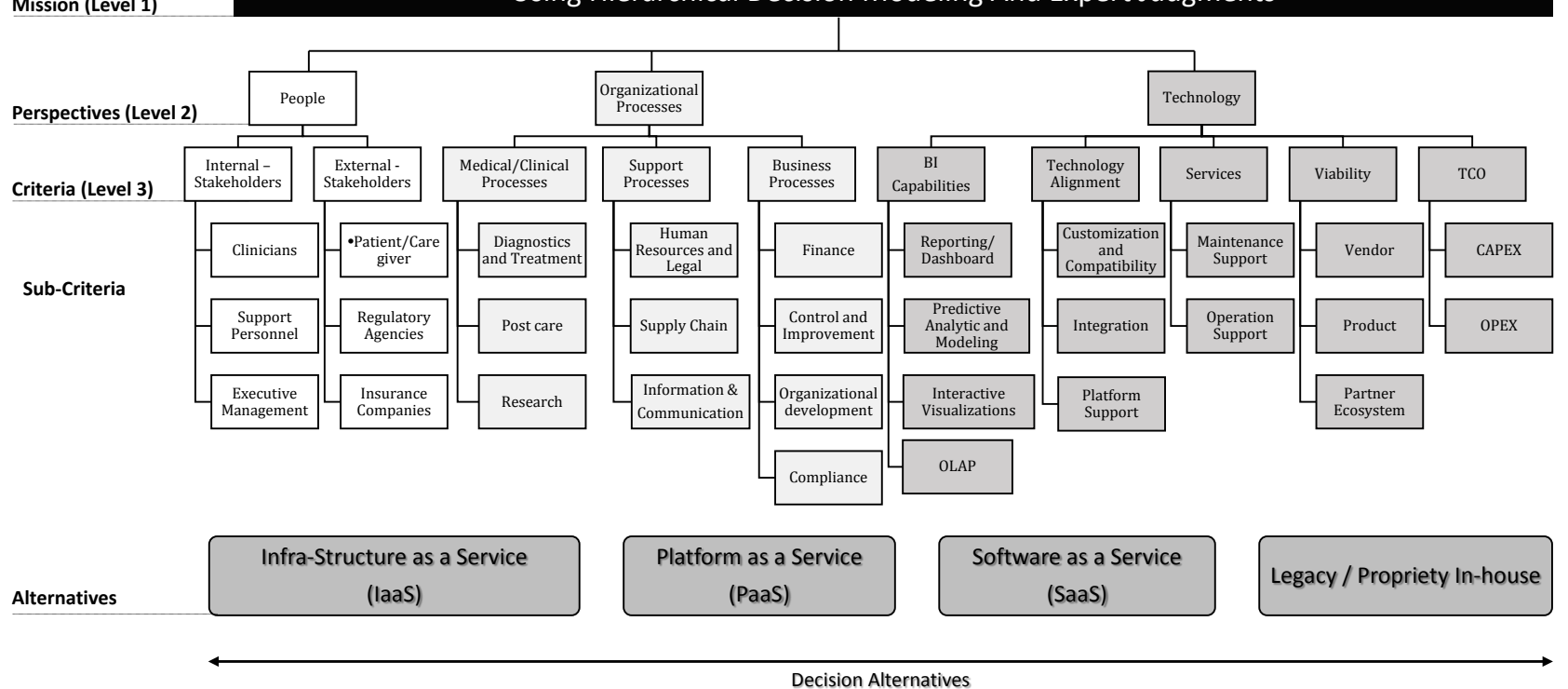

\section{Framework for the Assessment of Business Intelligence Platforms}

for Medium-to-Large Hospitals

Using Hierarchical Decision Modeling And Expert Judgments

Decision Alternatives

Figure 3. Technology assessment model. 
the $\mathrm{T}$ perspective but adding $\mathrm{O}$ and $\mathrm{P}$ perspectives may add new dimensions and insights. Adding the $\mathrm{T}$ perspective to the $\mathrm{O}$ perspective may bring more appreciation for the $\mathrm{P}$ perspective. One perspective may be of more value to one subject matter expert and may not to the other. Some perspectives are closely related, and some are not.

In this research $\mathrm{BI}$ assessment, three perspectives are People, Organizational process, and Technology (POT) are considered. Each perspective has numerous criteria as well as sub-criteria. Here is a brief definition of each perspective and criterion:

\subsection{People Perspective}

Organizations are made up of people. People are the stakeholders, stakeholders within the organization, referred "inside stakeholders," and outside the organization are "outside stakeholders." Probably the most difficult one, as each person has their intuition.

Internal Stakeholders: Individual or group of individuals who can affect and operate within the bounds of the organization.

Clinician: The expert utilizes a scientific knowledge base and is authorized to direct health care services to the patient.

Support Personnel: Individuals actively engage in improving the health of a patient.

Executive Management: This is defined as senior leadership responsible for improving the quality of care, efficiency, effectiveness, and lowering the cost of healthcare.

External Stakeholders: Can be defined as individuals or groups of individuals affected by (achieving) a business's purpose.

Patient/Caregiver: Individual waiting for the care to be received or have received. The individual who is sill or suffering from any disease.

Regulatory Agencies: Administrative entities outside the organization intend to monitor or influence the activity(es).

Insurance/HealthCare Insurance: is a system to make significant losses more affordable by pooling the risk of many individuals. The entity is sometimes known as an Insurance Company or insurance career.

\subsection{Organizational Process Perspective}

A business process is defined as the set of activities and tasks that, upon completion, will achieve an organizational goal.

Medical/Clinical Process: Comprises healthcare provider activities, addressing known or unknown health issues or activities performed by the healthcare provider for individual care with health issues.

Diagnostics and treatments: Identifying disease, condition, or injury from signs and symptoms. Treatment is patient care to combat disease determined by medical diagnosis. 
Post care/Aftercare (Treatment): Also referred to as treatment or care given to the individuals discharged from the hospital.

Research: Systematic study does include data collection, documentation, and analyzing the data. Action research is usually attached with hospitals.

Support processes: such processes are critical for the execution of management processes or operational processes or coordination between the two mentioned processes, necessary for improving healthcare quality and personal experience.

Human Resource (HR) and Legal: HR focus on improving the people experience. However, a legal department within the healthcare providers can assist in patient confidentiality, privacy issues, and informed consent.

Supply Chain: defined as the working relationship between a business and its suppliers (vendors). The supply chain is critical for the success of the business.

Information and Communication: It is an umbrella term covering all the digital technologies supporting communication of all the necessary information to improve the patient's health or better patient outcomes. Information sharing/communication is critical for improving healthcare quality and lowering costs.

Business Processes: Businesses are integrated through the business processes, a series of actions performed by the process players to achieve a common goal.

Finance: referred to as healthcare finance, consists of accounting and financial management for this research.

Controls and Improvement: Controls are critical for business processes and functions. It enhances the effectiveness of the processes.

Organization development: is referred to an organization's capabilities development for the research purpose. Organizational development is a constant effort.

Compliance: conforming to relevant regulations, laws, or policies. The level of compliance with rules and regulations is different at different levels of the organization.

\subsection{Technology Perspective}

The Technology perspective has overshadowed all the other views regarding technology assessment. It relates to the organization's technology environment, systems, data, and analytics.

BI Capabilities: Organizational and technological capabilities are considered while defining the BI capabilities. The most critical capabilities are flexibility, integration, data quality, and end-user access to BI.

Reporting/Dashboard: Reporting communicates the information gathered from various sources (clinical/financial/operational). However, the dashboard supports business analytic capabilities.

Predictive analytics and Modeling: Seek to predict future events or outcomes by analyzing patterns that are likely to forecast future results. 
Interactive Visualizations: Assists the user in their journey to explore the data using charts, colors, size, shape, and many visual objects representing attributes of the dataset under analysis.

Online Analytical Processing (OLAP): Enable the user to build a multidimensional database or also referred to as a cube.

Technology Alignment: For the current study, it is defined as an organization's active IT engagement to achieve the organization's objectives.

Customization and Compatibility: Necessary changes to software source code or configuration changes to meet the process requirements. Software compatibility is the software's ability to run on specific hardware or operating systems or integrate with other software applications. Compatibility of Hardware means the components that can be replaced because they have the same properties and mainly the same or similar design.

Integration: System integration is meant here. System integration is intended to connect various subsystems or independently running systems, bringing them together as one system.

Platform Support: back-end technology capabilities; they can be an individual system or group of various systems that run products.

Services: Service providers' and consumers' perspectives can be defined. IT services are meant here for the study. It can be referred to as the business and technical expertise to enable organizations to create, manage, and optimize information.

Maintenance Support: IT maintenance support is considered here. The maintenance of production systems is highly desirable as it will increase the process quality.

Operation Support: IT operation support is considered here. Operation support includes but is not limited to application and technical expert support.

Viability: It is the ability to function adequately-economic, technical, and user adoption perspectives before implementation.

Vendor: Individual or the company offering products (software, equipment, office supplies) or services (cloud service provider).

Product: The product is considered here a software product. The software product is an essential component to be considered for the business intelligence implementation.

Partner Ecosystem: The partner ecosystem is the partnerships a company has created to extend the digital footprint, resulting in a larger market share, which eventually translates into a competitive advantage.

Total Cost of Ownership (TC): The total cost of ownership (TCO) is defined as all relevant supply chain-related costs of doing business for a specific supplier, for a particular product or service.

Capital Expenditures: It is defined as the purchase of goods and services resulting in improved performance of the business in the future.

Operating Expenses: The operating expenses are the costs a business bears for 
the day-to-day expenses of running an operation.

\section{Conclusion}

For the first time, HDM methodology is applied for the assessment of BI, compared with traditional approaches, for example, cost-benefit analysis, forecasting, impact assessment, and policy studies or constructive technology assessment.

The framework presented here is comprehensive for mid-large hospitals. The current framework is validated by a team of experts, which will now be referred to as the model.

In the next step, the panel of experts will be selected to provide quantification, which will be applied for the categorization and relative ranking of factors that influence the selection of business intelligence alternatives. There are many factors, and understanding the relationship between factors and prioritizing the factors is at the core of HDM methodology. The potential experts with $10+$ years, their diverse backgrounds related to $\mathrm{BI}, \mathrm{HC}$, and Technology will provide valuable input to prioritize perspectives and factors.

\section{Assumption and Limitation}

The subject matter experts selected for the study have 10+ years of work experience. It is assumed that their opinion is representative of a large population of experts in the field of study. A 0 to 100 continuous scale can improve the results' accuracy compared to the seven stepped levels used in this study. However, more steps can be considered. We chose the seven steps at the unanimous request of the experts.

\section{Future Work}

Other worldviews such as small and medium-sized clinical practice, consulting companies, Insurance companies, or cloud service providers can be considered for future research. Each criterion can be expanded to multiple factors as the field evolves, definitions are clear, and independent attributes identified.

\section{Conflicts of Interest}

The authors declare no conflicts of interest regarding the publication of this paper.

\section{References}

Al Hadwer, A., Tavana, M., Gillis, D., \& Rezania, D. (2021). A Systematic Review of Organizational Factors Impacting Cloud-Based Technology Adoption Using Technology-Organization-Environment Framework. Internet of Things, 15, Article ID: 100407. https://doi.org/10.1016/j.iot.2021.100407

Alanazi, H., \& Daim, T. (2021). Health Technology Diffusion: Case of Remote Patient Monitoring (RPM) for the Care of Senior Population. Technology in Society, 66, Ar- 
ticle ID: 101662. https://doi.org/10.1016/j.techsoc.2021.101662

Ali, O., Shrestha, A., Soar, J., \& Wamba, S. F. (2018). Cloud Computing-Enabled Healthcare Opportunities, Issues, and Applications: A Systematic Review. International Journal of Information Management, 43, 146-158.

https://doi.org/10.1016/j.ijinfomgt.2018.07.009

Bach, M. P., ČEljo, A., \& Zoroja, J. (2016). Technology Acceptance Model for Business Intelligence Systems: Preliminary Research. Procedia Computer Science, 100, 995-1001. https://doi.org/10.1016/j.procs.2016.09.270

Banta, D. (2009). What Is Technology Assessment? International Journal of Technology Assessment in Health Care, 25, 7-9. https://doi.org/10.1017/S0266462309090333

Barham, H., \& Daim, T. (2020). The Use of Readiness Assessment for Big Data Projects. Sustainable Cities and Society, 60, Article ID: 102233. https://doi.org/10.1016/j.scs.2020.102233

Chang, Y. W., Hsu, P. Y., \& Shiau, W. L. (2013). An Empirical Study of Managers' Usage Intention in BI. Cognition, Technology \& Work, 16, 247-258.

https://doi.org/10.1007/s10111-013-0261-Z

Chaudhary, S. (2004). Management Factors for Strategic BI Success. In S. Mahesh, \& I. Raisinghani (Eds.), Business Intelligence in the Digital Economy (pp. 191-206). IGI Global. https://doi.org/10.4018/978-1-59140-206-0.ch010

Dayama, S. (2021, August 9). Legacy vs Cloud Based BI Tools. The Data School. https://dataschool.com/data-conversations/sdayama-legacyvscloud1/

de Carvalho, D., Rocha, R., Fernandes, V., \& Neves, S. (2016). Business Intelligence. In E. Desai (Ed.), Proceedings of the Ninth International $C^{*}$ Conference on Computer Science \& Software Engineering-C3S2E '16 (pp. 89-92). Association for Computing Machinery. https://doi.org/10.1145/2948992.2949011

Elena, C. (2011). Business Intelligence. Journal of Knowledge Management, Economics and Information Technology, 1, 1-12.

Guide, V. D., \& Pentico, D. (2003). A Hierarchical Decision Model for Re-Manufacturing and Re-Use. International Journal of Logistics Research and Applications, 6, 29-35. https://doi.org/10.1080/1367556031000063040

Hogaboam, L., \& Daim, T. (2018). Technology Adoption Potential of Medical Devices: The Case of Wearable Sensor Products for Pervasive Care in Neurosurgery and Orthopedics. Health Policy and Technology, 7, 409-419. https://doi.org/10.1016/j.hlpt.2018.10.011

Hou, F. (2016). A Hierarchical Decision Model Based on Pairwise Comparisons 1. Fundamenta Informaticae, 144, 333-348. https://doi.org/10.3233/FI-2016-1339

IBM Cloud Education (2021, September 16). IaaS vs. PaaS vs. SaaS. IBM. https://www.ibm.com/cloud/learn/iaas-paas-saas

Isik, O. (2009). Business Intelligence Success: An Empirical Evaluation of the Role of BI Capabilities and Organization's Decision Environment. In AMCIS 2009 Doctoral Consortium (Article No. 13). http://aisel.aisnet.org/amcis2009 dc/13

Kakkanatt, C., Benigno, M., Jackson, V. M., Huang, P. L., \& Ng, K. (2018). Curating and Integrating User-Generated Health Data from Multiple Sources to Support Healthcare Analytics. IBM Journal of Research and Development, 62, 2:1-2:7. https://doi.org/10.1147/JRD.2017.2756742

Kao, H. Y., Yu, M. C., Masud, M., Wu, W. H., Chen, L. J., \& Wu, Y. C. J. (2016). Design and Evaluation of Hospital-Based Business Intelligence System (HBIS): A Foundation for Design Science Research Methodology. Computers in Human Behavior, 62, 495-505. 
https://doi.org/10.1016/j.chb.2016.04.021

Kocaoglu, D. F. (1983). A Participative Approach to Program Evaluation. IEEE Transactions on Engineering Management, EM-30, 112-118. https://doi.org/10.1109/TEM.1983.6448602

Kumar Sharma, D., Sreenivasa Chakravarthi, D., Ara Shaikh, A., al Ayub Ahmed, A., Jaiswal, S., \& Naved, M. (2021). The Aspect of Vast Data Management Problem in Healthcare Sector and Implementation of Cloud Computing Technique. Materials Today: Proceedings (In Press). https://doi.org/10.1016/j.matpr.2021.07.388

Lal, P., \& Bharadwaj, S. S. (2016). Understanding The impact of Cloud-Based Services Adoption on Organizational Flexibility. Journal of Enterprise Information Management, 29, 566-588. https://doi.org/10.1108/JEIM-04-2015-0028

Lingga, M. (2000). Developing a Hierarchical Decision Model to Evaluate Nuclear Power Plant Alternative Siting Technologies. PDX Scholar. https://doi.org/10.15760/etd.2938

Linstone, H. A. (1981). The Multiple Perspective Concept. Technological Forecasting and Social Change, 20, 275-325. https://doi.org/10.1016/0040-1625(81)90062-7

Luhn, H. P. (1958). A Business Intelligence System. IBM Journal of Research and Development, 2, 314-319. https://doi.org/10.1147/rd.24.0314

Mettler, T., \& Vimarlund, V. (2009). Understanding Business Intelligence in the Context of Healthcare. Health Informatics Journal, 15, 254-264.

https://doi.org/10.1177/1460458209337446

Microsoft (2021). Public Cloud vs Private Cloud vs Hybrid Cloud. Microsoft Azure. https://azure.microsoft.com/en-us/overview/what-are-private-public-hybrid-clouds/\#d eployment-options

Mudavadi, C., Hogaboam, L., \& Daim, T. U. (2016). A Hierarchical Decision Model (HDM) for Exploring the Adoption of Electronic Health Records. In 2016 Portland International Conference on Management of Engineering and Technology (PICMET) (pp. 2770-2781). Institute of Electrical and Electronics Engineers. https://doi.org/10.1109/PICMET.2016.7806627

Olszak, C. M., \& Ziemba, E. (2007). Approach to Building and Implementing Business Intelligence Systems. Interdisciplinary Journal of Information, Knowledge, and Management, 2, 135-148. https://doi.org/10.28945/105

Pereira, C. G., Lavoie, J. R., Garces, E., Basso, F., Dabić, M., Porto, G. S., \& Daim, T. (2019). Forecasting of Emerging Therapeutic Monoclonal Antibodies Patents Based on a Decision Model. Technological Forecasting and Social Change, 139, 185-199. https://doi.org/10.1016/j.techfore.2018.11.002

Precedence Research. (2021, August 26). Healthcare Cloud Computing Market Size, Report 2020 to 2027.

https://www.precedenceresearch.com/healthcare-cloud-computing-market

RBC Capital Markets (2021, September 10). The Healthcare Data Explosion. https://www.rbccm.com/en/gib/healthcare/episode/the healthcare data explosion

Research and Markets (2020, August 21). Cloud Computing Industry to Grow from $\$ 371.4$ Billion in 2020 to $\$ 832.1$ Billion by 2025 , at a CAGR of $17.5 \%$. GlobeNewswire News Room.

https://www.globenewswire.com/news-release/2020/08/21/2081841/0/en/Cloud-Comp ut-

ing-Industry-to-Grow-from-371-4-Billion-in-2020-to-832-1-Billion-by-2025-at-a-CAG R-of-17-5.html

Schmidt, K., Aumann, I., Hollander, I., Damm, K., \& von der Schulenburg, J. M. G. 
(2015). Applying the Analytic Hierarchy Process in Healthcare Research: A Systematic Literature Review and Evaluation of Reporting. BMC Medical Informatics and Decision Making, 15, Article No. 112. https://doi.org/10.1186/s12911-015-0234-7

Serumaga-Zake, P. A. (2017). The Role of User Satisfaction in Implementing a Business Intelligence System. SA Journal of Information Management, 19, Article No. a736. https://doi.org/10.4102/sajim.v19i1.736

Sharma, R., Mithas, S., \& Kankanhalli, A. (2014). Transforming Decision-Making Processes: A Research Agenda for Understanding the Impact of Business Analytics on Organisations. European Journal of Information Systems, 23, 433-441. https://doi.org/10.1057/ejis.2014.17

Sheikh, N. J., Kim, K., \& Kocaoglu, D. F. (2016). Use of Hierarchical Decision Modeling to Select Target Markets for a New Personal Healthcare Device. Health Policy and Technology, 5, 99-112. https://doi.org/10.1016/j.hlpt.2015.12.001

Sheikh, N. J., Kocaoglu, D. F., \& Lutzenhiser, L. (2016). Social and Political Impacts of Renewable Energy: Literature Review. Technological Forecasting and Social Change, 108, 102-110. https://doi.org/10.1016/j.techfore.2016.04.022

Simonson, S. B. (2020, May 21). How Business Intelligence Delivers Financial Insight to Outreach Labs. Change Healthcare.

https://www.changehealthcare.com/insights/business-intelligence-delivers-financial-in sight-to-labs

Son, W., \& Sheikh, N. J. (2018). Assessment of Electronic Authentication Policies using Multi-Stakeholder Multi-Criteria Hierarchical Decision Modeling. In 2018 Portland International Conference on Management of Engineering and Technology (PICMET) (pp. 1-11). Institute of Electrical and Electronics Engineers. https://doi.org/10.23919/PICMET.2018.8481798

Taleb, N., \& Mohamed, E. A. (2020). Cloud Computing Trends: A Literature Review. Academic Journal of Interdisciplinary Studies, 9, 91. https://doi.org/10.36941/ajis-2020-0008

Zheng, G., Zhang, C., \& Li, L. (2014). Bringing Business Intelligence to Health Information Technology Curriculum. In Proceedings of the 45th ACM Technical Symposium on Computer Science Education (pp. 205-210). Association for Computing Machinery. https://doi.org/10.1145/2538862.2538935 